\title{
Advertising and production of a seasonal good for a heterogeneous market
}

\author{
Daniela Favaretto ${ }^{1}$, Bruno Viscolani ${ }^{2}{ }^{\star}$ \\ 1 Department of Applied Mathematics, University "Ca' Foscari" of Venice, \\ Dorsoduro 3825/E, I-30123 Venezia, Italy, e-mail: favaret@unive.it \\ 2 Department of Pure and Applied Mathematics, University of Padua, \\ Via Trieste 63, I-35121 Padova, Italy, e-mail: viscolani@math.unipd.it \\ Received: date / Revised version: date
}

\begin{abstract}
We bring some concepts from market segmentation, which is a fundamental topic of marketing theory and practice, into the statement of an advertising and production problem for a seasonal product with NerloveArrow's linear goodwill dynamics. We consider two kinds of situations. In the first one the advertising process can reach selectively each segment. In the second one one advertising medium is available which has a known effectiveness spectrum for a non-trivial set of segments. In both cases we solve, using the Pontryagin's Maximum Principle conditions, the optimal control problems in which goodwill productivity of advertising is concave and good production cost is convex. Two special cases are discussed in detail.
\end{abstract}

Keywords: Marketing; Advertising; Optimal Control.

Mathematics Subject Classification: 90B60; 49J15.

\section{Introduction}

The purpose of this paper is to analyse the consequences of a market segmentation in the optimal advertising and production decisions of a firm, when a seasonal good is concerned. We study a couple of finite horizon and

* Supported by the Italian Ministry of University and Research, the University of Padua and the University of Venice. 
deterministic optimal control problems in order to obtain optimal policies under two contrasting assumptions on advertising media availability.

Although the infinite horizon formulation is the natural setting for the analysis of some dynamic optimization problems in advertising, as for instance in the recent papers (Jørgensen et al., 2001), (Weber, 2005), (Grosset and Viscolani, 2008), there are special situations for which a finite horizon formulation is obviously needed. Examples of this case are advertising for an event, as in (Jørgensen et al., 2006), introducing a new product, as in (Buratto et al., 2006a), and advertising for a seasonal product, as in (Favaretto and Viscolani, 2004).

Market segmentation is a fundamental topic of marketing theory and practice (Kotler, 2003, Chapter 10) and it recalls the use of multiple advertising media, necessary to reach audiences with different media habits (Kotler and Trias de Bes, 2003, p. 11). Nevertheless, market segmentation is not well represented in mathematical models in advertising, and in particular in the literature on optimal control models (Feichtinger et al., 1994; Jørgensen and Zaccour, 2004). Buratto, Grosset and Viscolani have brought some market segmentation concepts into models concerning the advertising process while introducing a new product in a market (Buratto et al., 2006a,b), and the advertising process for sales over an infinite horizon (Grosset and Viscolani, 2008). Here we want to do the same in the context of an advertising and production problem for a seasonal product with Nerlove-Arrow's linear goodwill dynamics (Nerlove and Arrow, 1962), which has been discussed widely under the usual assumption of a homogeneous market (Favaretto et al., 1996; Favaretto and Viscolani, 1997, 1999, 2004).

We consider the problem of determining an optimal advertising policy and production quantity, in order to maximize the net profit. We analyse two kinds of situations. In the first one, the advertising process can reach selectively each segment. In the second one, one advertising medium is available which has a known effectiveness spectrum for a non-trivial set of segments. In both cases we formulate the general optimal control problem and we give necessary and sufficient conditions for optimality. In particular we study the optimal control problems in which goodwill productivity of advertising is concave, and good production cost is convex.

Here is the outline of the paper. In Section 2 we consider a heterogeneous market and we assume that each segment has a stock of goodwill. We describe the goodwill evolution in the different segments as governed by independent ordinary differential equations. In Section 3 we consider a firm which produces, sells and advertises a seasonal product and we assume that the advertising process can reach selectively each segment. We solve the general advertising and production problem and we examine the special cases in which the production costs are quadratic, whereas the advertising 
productivity is represented by a square root or a logarithmic function. In Section 4 we solve the analogous problem under the assumption that only one advertising medium is available and that it has a known effectiveness over the segment set.

\section{Seasonal good: goodwill evolution in a segmented market}

Let the consumers population be partitioned into groups (segments), each one specified by the value $a \in A$ of a suitable parameter (segmentation attribute). Typical finite segmentations are obtained using such attributes as region, age, gender, occupation, generation, lifestyle, occasions, ... (see Kotler, 2003, p. 288).

Let $G_{a}(t)$ represent the stock of goodwill of the product at time $t$, for the (consumers in the) a segment. We refer to the definition of goodwill given by Nerlove and Arrow (1962) to describe the variable which summarizes the effects of present and past advertising on the demand; the goodwill needs an advertising effort to increase, while it is subject to a spontaneous decay. Here we assume that the goodwill evolution satisfies the set of independent ordinary differential equations

$$
\dot{G}_{a}(t)=w_{a}(t)-\delta_{a} G_{a}(t), \quad a \in A,
$$

where $\delta_{a}>0$ represents the goodwill depreciation rate for the members of the consumers group $a$ and $w_{a}(t)$ is the effective advertising intensity at time $t$ directed to that same group. For each fixed value of the parameter $a \in A$, i.e. for each segment, the dynamics of the goodwill given by (1) is essentially the same as the one proposed in (Nerlove and Arrow, 1962). Here, consistent with the assumption of distinct goodwill variables for different market segments, we further assume that both the advertising intensity and the goodwill decay parameter may depend on the attribute value $a$. The vector of the effective advertising intensities $\left(w_{a}(t)\right)_{a \in A}$ is the result of using one or several advertising media. Using several media, even as many as the market segments, may be necessary to reach consumers with different media habits and may be highly expensive (Kotler and Trias de Bes, 2003, p. 11). A company which produces digital cameras may reach people with ads on a certain new camera through television (high reach, low audience selectivity), or through magazines (high geographic and demographic selectivity), radio, newspapers, ... (Kotler, 2003, p. 601). Whenever multiple media affect the goodwill evolution of a market segment, a synergy effect may be present (Naik and Raman, 2003), which should be represented in the model. For the sake of simplicity, we consider two extreme situations. First, in Section 3, we study the case of maximum audience selectivity, in which one advertising medium is available for each market segment, and that medium reaches only the consumers of the associated segment. Second, in Section 4, we study 
the case of no audience selectivity, in which a single medium is available to the company: it reaches all the market segments, although with different advertising effectiveness.

We consider a firm which produces (or purchases), advertises and sells a seasonal product. Production and sales take place in two consecutive time intervals; we denote by $[0,1]$ the sales one. We want to determine the quantity of good to have available at time 0 and an advertising policy in the sales interval, in order to maximize the net profit. As the season is a short time horizon, we consider undiscounted costs and revenue. The values of the goodwill components at the initial time 0 are known data:

$$
G_{a}(0)=G_{a}^{0} \geq 0, \quad a \in A
$$

The demand intensity depends linearly on the goodwill so that the sales quantity until time $t$ is

$$
x(t)=\int_{0}^{t} \sum_{a \in A} \beta_{a} G_{a}(s) d s .
$$

The parameter $\beta_{a}>0$ is the marginal demand of goodwill in segment $a$ : its value depends on the dimension of the segment, i.e. number of potential consumers in it, and on the interest of those consumers to the product. The constant product price is 1 , so that the total revenue from sales coincides with $x(1)$. Let $c(\cdot)$ be the production cost function of the seasonal good, a nonnegative, increasing and strictly convex function, with $c(0)=0$; we assume it is continuously differentiable, so that $c^{\prime}(\cdot)>0$ is strictly increasing. In a deterministic setting, the firm produces exactly the demanded quantity $x(1)$, so that the total production cost is $c(x(1))$. We observe that if the manufacturer does not advertise towards any segment, $u_{a}(t) \equiv 0, a \in A$, then $G_{a}(t)=G_{a}^{0} e^{-\delta_{a} t}$ and

$$
x(1)=x_{\min }=\sum_{a \in A} \frac{\beta_{a} G_{a}^{0}}{\delta_{a}}\left(1-e^{-\delta_{a}}\right) .
$$

In order to avoid trivial situations we assume that marginal production cost at the minimum quantity $x_{\min }$ is less than price,

$$
c^{\prime}\left(x_{\min }\right)<1
$$

otherwise it would not be convenient for the firm to advertise, nor to produce any unit of the good more than $x_{\min }$. 


\section{Advertising problem with total segment-resolution}

We assume that the goodwill evolution is driven by the media advertising efforts $u_{a}(t) \geq 0$ (the control functions, measured in cost per unit time) in such a way that the effective advertising intensity at time $t$ directed to segment $a$ is

$$
w_{a}(t)=\varphi_{a}\left(u_{a}(t)\right),
$$

where the function $\varphi_{a}(\cdot)$, the productivity of the medium advertising effort, is a nonnegative, increasing and strictly concave function; we assume it is continuously differentiable, so that $\varphi_{a}^{\prime}(\cdot)>0$ is strictly decreasing and hence invertible. Moreover we require that $\lim _{u_{a} \rightarrow+\infty} \varphi_{a}^{\prime}\left(u_{a}\right)=0$ and we will distinguish the possibility that the marginal productivity of the medium advertising effort is bounded at $0, \varphi_{a}^{\prime}(0) \in(0,+\infty)$, from the alternative possibility that it is unbounded, $\lim _{u_{a} \rightarrow 0} \varphi_{a}^{\prime}\left(u_{a}\right)=+\infty$.

Assuming that equation (1) describes the dynamics of the system, with the effective advertising effort specified by (6), amounts to assuming that the firm may control an advertising process, with such a high segmentresolution, as to be able to reach each segment with the desired intensity. This is the total segment-resolution assumption, which is characteristic of micromarketing (see Kotler, 2003, p. 279).

The advertising and production problem of finding some media advertising effort functions $u_{a}(t)$ in order to maximize the firm profit is

$$
\begin{aligned}
& \text { maximize } J(u)=x(1)-\int_{0}^{1} \sum_{a \in A} u_{a}(t) d t-c(x(1)) \\
& \text { subject to } \dot{G}_{a}(t)=\varphi_{a}\left(u_{a}(t)\right)-\delta_{a} G_{a}(t), \quad a \in A \text {, } \\
& \dot{x}(t)=\sum_{a \in A} \beta_{a} G_{a}(t), \\
& G_{a}(0)=G_{a}^{0}, \quad a \in A, \\
& x(0)=0 \text {, } \\
& u_{a}(t) \geq 0, \quad a \in A \text {. }
\end{aligned}
$$

Here, the control $u$ is the vector of components $u_{a}, a \in A$. The firm profit is the difference between the revenue from sales and the total advertising and production costs.

\subsection{Optimal advertising policy}

In this Section we provide a characterization of a possible optimal control for the advertising and production problem and prove that the unique solution to such conditions is indeed optimal. 
Theorem 1. Let $(u(t), G(t), x(t))=\left(\left\{u_{a}(t)\right\}_{a \in A},\left\{G_{a}(t)\right\}_{a \in A}, x(t)\right)$ be an optimal solution, then the a-component of the optimal control is the continuous function

$$
u_{a}(t)= \begin{cases}\psi_{a}\left(\frac{\delta_{a}}{\bar{\mu} \beta_{a}}\left(1-e^{-\delta_{a}(1-t)}\right)^{-1}\right), & 0 \leq t<\tau_{a}, \\ 0, & \tau_{a} \leq t \leq 1,\end{cases}
$$

where $\psi_{a}(\cdot)$ is the inverse function of the marginal advertising productivity at segment $a, \varphi_{a}^{\prime}(\cdot)$, moreover

$$
\bar{\mu}=1-c^{\prime}(x(1))>0,
$$

and

- if $\lim _{u_{a} \rightarrow 0} \varphi_{a}^{\prime}\left(u_{a}\right)=+\infty$, then $\tau_{a}=1$,

- if $\varphi_{a}^{\prime}(0) \in(0,+\infty)$, then $\tau_{a}=\max \left\{0, \tau_{a}^{\prime}\right\}$, where

$$
\tau_{a}^{\prime}=1+\frac{1}{\delta_{a}} \ln \left(1-\frac{\delta_{a}}{\bar{\mu} \beta_{a} \varphi_{a}^{\prime}(0)}\right) .
$$

There exists a unique candidate optimal solution.

Proof. The problem Hamiltonian is

$$
\begin{aligned}
H(G, x, u, \lambda, \mu, t)= & \sum_{a \in A}\left\{-\lambda_{0} u_{a}+\lambda_{a} \varphi_{a}\left(u_{a}\right)\right\} \\
& +\sum_{a \in A}\left\{-\lambda_{a} \delta_{a}+\mu \beta_{a}\right\} G_{a},
\end{aligned}
$$

which is a continuously differentiable function of $(G, x, u)$.

From the Pontryagin Maximum Principle conditions (see Seierstad and Sydsaeter, 1987, p. 85) we obtain, for some constants $\lambda_{0}, \eta_{a}(a \in A), \mu_{1} \in \Re$ and functions $\lambda_{a}(t)(a \in A), \mu(t)$, that

i) $\left(\lambda_{0},\left\{\eta_{a}\right\}_{a \in A}, \mu_{1}\right) \neq 0$;

ii) for all $a$ and all $t, u_{a}(t)$ maximizes

$$
-\lambda_{0} u_{a}+\lambda_{a}(t) \varphi_{a}\left(u_{a}\right), \quad u_{a} \geq 0,
$$

which is a concave function of $u_{a}$, as far as $\lambda_{a}(t) \geq 0$;

iii) for all $a$ and virtually all $t$,

$$
\begin{aligned}
& \dot{\lambda}_{a}(t)=\delta_{a} \lambda_{a}(t)-\beta_{a} \mu(t), \\
& \dot{\mu}(t)=0 ;
\end{aligned}
$$

iv) $\lambda_{0} \in\{0,1\}$; 
v) for all $a$,

$$
\lambda_{a}(1)=\eta_{a}=0,
$$

and finally

$$
\mu(1)=\lambda_{0}\left(1-c^{\prime}(x(1))\right)+\mu_{1}, \quad \mu_{1}=0 .
$$

It is easy to observe that $\lambda_{0}=1$ for all solutions and that an optimal control $u_{a}(t)$ must satisfy the following conditions.

If $\lambda_{a}(t) \leq 0$ then $u_{a}(t)=0$, whereas if $\lambda_{a}(t)>0$ we have to distinguish between bounded and unbounded marginal productivity of the medium advertising effort. In the unbounded case, $\lim _{u_{a} \rightarrow 0} \varphi_{a}^{\prime}\left(u_{a}\right)=+\infty$, we have

$$
u_{a}(t)=\psi_{a}\left(1 / \lambda_{a}(t)\right),
$$

where $\psi_{a}(\cdot)$ is the inverse function of the marginal advertising productivity at segment $a, \varphi_{a}^{\prime}(\cdot)$.

In the bounded case, $\varphi_{a}^{\prime}(0) \in(0,+\infty)$, we have

$$
u_{a}(t)= \begin{cases}\psi_{a}\left(1 / \lambda_{a}(t)\right), & \lambda_{a}(t)>1 / \varphi_{a}^{\prime}(0), \\ 0, & \lambda_{a}(t) \leq 1 / \varphi_{a}^{\prime}(0) .\end{cases}
$$

We recall that we have assumed $\varphi_{a}(\cdot)$ strictly concave, so that $\varphi_{a}^{\prime}(\cdot)$ is invertible and the equations (12) and (13) make sense.

From the adjoint equations (iii) and the transversality conditions (v), we obtain that

$$
\mu(t) \equiv \bar{\mu}=1-c^{\prime}(x(1))
$$

and

$$
\lambda_{a}(t)=\frac{\bar{\mu} \beta_{a}}{\delta_{a}}\left(1-e^{-\delta_{a}(1-t)}\right) .
$$

Now, if $\bar{\mu} \leq 0$, then $\lambda_{a}(t)=0$, for all $a$ and $t$, hence $u_{a}(t)=0$, $t \in[0,1]$, and finally $x(1)=x_{\min }$, so that $1-c^{\prime}(x(1))>0$, because of the non-triviality condition (5). As $\bar{\mu} \leq 0$, this contradicts condition (14).

Then $\bar{\mu}>0$ and condition (9) must hold. It follows that the optimal advertising effort $u_{a}(t)$ decreases as time goes by, for all segments $a \in A$. Moreover,

- if the marginal productivity of advertising effort towards segment $a$ is bounded at $0, \varphi_{a}^{\prime}(0) \in(0,+\infty)$, then

- either $u_{a}(0)=0$, and $u_{a}(t)=0$, for all $t \in[0,1]$;

- or $u_{a}(0)>0$, and $u_{a}(t)=0, t \in\left[\tau_{a}^{\prime}, 1\right]$, for some critical time $\tau_{a}^{\prime} \in(0,1)$ such that $\lambda_{a}\left(\tau_{a}^{\prime}\right)=1 / \varphi_{a}^{\prime}(0)$, which gives condition (10). 
- if the marginal productivity of the medium advertising effort is unbounded, $\lim _{u_{a} \rightarrow 0} \varphi_{a}^{\prime}\left(u_{a}\right)=+\infty$, then $u_{a}(t)>0$ for all $t<1$ and

$$
\lim _{t \rightarrow 1} u_{a}(t)=0 \text {. }
$$

Finally, we observe that, for all $a \in A$ and $t \leq 1$, the control $u_{a}(t)$ is a monotonically increasing and continuous function of $\bar{\mu}$ on the interval $[0,+\infty)$, and so are the goodwill components $G_{a}(t)$ and the total sales $x(1)$. The right hand side of the transversality equation (14) is a continuous and monotonically decreasing function of $\bar{\mu}$, moreover it is strictly positive at $\bar{\mu}=0$ where $x(1)=x_{\min }$, because of (5), therefore there exists a unique solution to equation (14).

Theorem 2. If $(u(t), G(t), x(t), \lambda(t), \bar{\mu})$ is a solution to the necessary conditions, then it is the optimal solution.

Proof. If $(u(t), G(t), x(t), \lambda(t), \bar{\mu})$ is a solution to the necessary conditions, then $\bar{\mu}>0$ and $\lambda_{a}(t)>0$ for all $a \in A$, so that the Hamiltonian is concave in $(G, x, u)$. Now, all the assumptions of the Mangasarian sufficiency theorem (see Seierstad and Sydsaeter, 1987, p. 105) hold, so that the unique solution to the necessary conditions is the optimal solution.

We observe that the optimal advertising effort $u_{a}(t)$ decreases as time goes by, for all segments $a \in A$. Moreover, it is optimal to advertise, at low levels, near the season end if and only if the marginal productivity of the medium advertising effort is unbounded, otherwise even very low advertising levels must be considered as not convenient, because there is not enough time to obtain a good revenue. It may even occur that no advertising at any time and for any segment is optimal, i.e. that $\tau_{a}=0$ for all $a \in A$.

For any two segments $a, b \in A$, at which (i) the marginal advertising productivity is bounded, $\varphi_{a}^{\prime}(0), \varphi_{b}^{\prime}(0) \in(0,+\infty)$, (ii) the decay parameters are the same, $\delta_{a}=\delta_{b}$, and (iii) the optimal controls are not $0, \tau_{a}, \tau_{b}>0$, we have that the advertising stopping times satisfy

$$
\tau_{a} \geq \tau_{b} \quad \Leftrightarrow \quad \beta_{a} \varphi_{a}^{\prime}(0) \geq \beta_{b} \varphi_{b}^{\prime}(0)
$$

Therefore, in such a special case, the optimal policy requires to advertise for a longer time in segments where effectiveness of advertising expenditure is higher.

\subsection{Square root advertising productivity and quadratic production costs}

Here and in all the following examples we assume a quadratic production cost function

$$
c(x)=c_{1} x+\frac{1}{2} c_{2} x^{2},
$$


with $c_{1} \geq 0$ and $c_{2}>0$, so that the marginal production cost is $c^{\prime}(x)=$ $c_{1}+c_{2} x$.

Let us consider the special case of square root productivity of media advertising efforts

$$
\varphi_{a}\left(u_{a}\right)=\gamma_{a} \sqrt{u_{a}}, \quad a \in A
$$

where $\gamma_{a}>0, a \in A$, is a parameter which affects positively the (unbounded) marginal productivity of the medium advertising effort directed to the segment $a$.

The inverse of the function $\varphi_{a}^{\prime}$ is $\psi_{a}(y)=\left(\gamma_{a} / 2 y\right)^{2}, y>0$; the nontriviality condition (5) reads

$$
1-c_{1}-c_{2} \sum_{a \in A} \frac{\beta_{a} G_{a}^{0}}{\delta_{a}}\left(1-e^{-\delta_{a}}\right)>0
$$

and the goodwill motion equations are

$$
\dot{G}_{a}(t)=\gamma_{a} \sqrt{u_{a}(t)}-\delta_{a} G_{a}(t), \quad a \in A
$$

We obtain that there exists the unique set of optimal advertising efforts

$$
u_{a}(t)=\frac{\bar{\mu}^{2}}{4}\left(\frac{\beta_{a} \gamma_{a}}{\delta_{a}}\right)^{2}\left(1-e^{-\delta_{a}(1-t)}\right)^{2},
$$

where the parameter $\bar{\mu}>0$ is determined by the transversality condition (9), which is

$$
\bar{\mu}=1-c_{1}-c_{2} x(1) .
$$

The firm will advertise optimally towards all segments at a strictly decreasing and positive level at all times $t<1$. Moreover the advertising effort vanishes at the final time $t=1$.

We observe that for any two segments $a, b \in A$, the advertising effort ratio $u_{a}(t) / u_{b}(t)$ is proportional to $\left(1-e^{-\delta_{a}(1-t)}\right)^{2} /\left(1-e^{-\delta_{b}(1-t)}\right)^{2}$ at all times $t<1$. In particular, if the decay parameters in the two segments are the same $\delta_{a}=\delta_{b}$, then the advertising effort ratio is constant and

$$
u_{a}(t) \geq u_{b}(t) \Leftrightarrow \beta_{a} \gamma_{a} \geq \beta_{b} \gamma_{b}
$$

Quite naturally, the optimal policy requires a higher advertising effort towards segments where effectiveness of advertising expenditure is higher. 


\subsection{Logarithmic advertising productivity}

Let us consider the special case of logarithmic productivity of media advertising efforts

$$
\varphi_{a}\left(u_{a}\right)=\gamma_{a} \ln \left(1+u_{a}\right), \quad a \in A,
$$

similar to that proposed by Sethi (1975) and used also in Jørgensen (1982), where $\gamma_{a}>0, a \in A$, is a parameter which affects positively the (bounded) marginal productivity of the medium advertising effort directed to the segment $a$.

Now, the inverse of the function $\varphi_{a}^{\prime}$ is $\psi_{a}(y)=-1+\gamma_{a} / y, y \in\left(0, \gamma_{a}\right]$. The non-triviality condition (5) is again (20) and the goodwill motion equations are

$$
\dot{G}_{a}(t)=\gamma_{a} \ln \left(1+u_{a}(t)\right)-\delta_{a} G_{a}(t), \quad a \in A
$$

The unique optimal advertising effort for segment $a$ is

$$
u_{a}(t)=\left[-1+\frac{\bar{\mu} \beta_{a} \gamma_{a}}{\delta_{a}}\left(1-e^{-\delta_{a}(1-t)}\right)\right]^{+},
$$

where $[\xi]^{+}=\max \{0, \xi\}$ and the parameter $\bar{\mu}>0$ is determined by the transversality condition (9), which reads as in (23). Here we have that $\varphi_{a}^{\prime}(0)=\gamma_{a}<+\infty$, so that the critical time $\tau_{a}^{\prime}$ from (10) is

$$
\tau_{a}^{\prime}=1+\frac{1}{\delta_{a}} \ln \left(1-\frac{\delta_{a}}{\bar{\mu} \beta_{a} \gamma_{a}}\right)<1 .
$$

If $\tau_{a}^{\prime}>0$, the firm will advertise optimally towards segment $a$ at a strictly decreasing and positive level until the time $\tau_{a}=\tau_{a}^{\prime}$, when the advertising effort is $0, u_{a}\left(\tau_{a}\right)=0$, and it remains 0 afterwards.

For any two segments $a, b \in A$, at which the decay parameters are the same, $\delta_{a}=\delta_{b}$, and $\tau_{a}, \tau_{b}>0$, we observe the relation between advertising stopping times and segment effectiveness of advertising expenditure:

$$
\tau_{a} \geq \tau_{b} \quad \Leftrightarrow \quad \beta_{a} \gamma_{a} \geq \beta_{b} \gamma_{b} .
$$

\section{Advertising problem with wide spectrum advertising}

Let us consider the situation in which the company has to use an advertising medium which reaches several segments with variable effectiveness, instead of using a set of segment-specific media. Let $u(t) \geq 0$ be the medium advertising effort (measured in cost per unit time) and let the effective advertising intensity at time $t$ directed to segment $a$ be

$$
w_{a}(t)=\varphi_{a}(u(t)), \quad a \in A .
$$


In particular we assume here that

$$
\varphi_{a}(u)=\gamma_{a} \varphi(u), \quad a \in A,
$$

for some segment specific parameters $\gamma_{a} \geq 0, a \in A$, such that $\sum_{a \in A} \gamma_{a}=$ 1 , and a function $\varphi(\cdot)$, so that the goodwill motion equations (1) look as follows:

$$
\dot{G}_{a}(t)=\gamma_{a} \varphi(u(t))-\delta_{a} G_{a}(t), \quad a \in A .
$$

The function $\varphi(\cdot)$, the productivity of the medium advertising effort, is a nonnegative, increasing and strictly concave function; furthermore it is continuously differentiable, so that $\varphi^{\prime}(\cdot)>0$ is strictly decreasing and hence invertible. We call $\left(\gamma_{a}\right)_{a \in A}$ the medium (segment-)spectrum. Its components, $\gamma_{a}, a \in A$, provide the different relative effectiveness of the advertising medium on the market segments.

The advertising and production problem requires to find a medium advertising effort $u(t) \geq 0$, in order to maximize the firm profit given by the functional

$$
J(u)=x(1)-\int_{0}^{1} u(t) d t-c(x(1)),
$$

under the conditions represented by the goodwill and sales motion equations (32) and (3) and the initial conditions (2).

Theorem 3. There exists a unique optimal solution $(u(t), G(t), x(t))=$ $\left(u(t),\left\{G_{a}(t)\right\}_{a \in A}, x(t)\right)$; the control is the continuous function

$$
u(t)= \begin{cases}\psi\left(\left[\bar{\mu} \sum_{a \in A} \frac{\beta_{a} \gamma_{a}}{\delta_{a}}\left(1-e^{-\delta_{a}(1-t)}\right)\right]^{-1}\right), & 0 \leq t<\tau, \\ 0, & \tau \leq t \leq 1\end{cases}
$$

where $\psi(\cdot)$ is the inverse function of the marginal advertising productivity $\varphi^{\prime}(\cdot)$, moreover

$$
\bar{\mu}=1-c^{\prime}(x(1))>0
$$

and

- if $\lim _{u \rightarrow 0} \varphi^{\prime}(u)=+\infty$, then $\tau=1$,

- if $\varphi^{\prime}(0) \in(0,+\infty)$, then $\tau=\max \left\{0, \tau^{\prime}\right\}$, where $\tau^{\prime}$ is the unique solution of the equation

$$
\sum_{a \in A} \frac{\beta_{a} \gamma_{a}}{\delta_{a}}\left(1-e^{-\delta_{a}(1-t)}\right)=\frac{1}{\bar{\mu} \varphi^{\prime}(0)}
$$


We can prove Theorem 3 along the same lines as for the pair of Theorems 1 and 2 and we observe that the optimal activation level $u(t)$ has the same qualitative features of the segment optimal controls $u_{a}(t)$ of Section 3.1. In the case that the goodwill decay parameter is the same for all segments, $\delta_{a}=\delta, a \in A$, the problem degenerates and the optimal advertising level (34) is formally the same as (8), with the parameter substitutions $\delta, \sum_{a \in A} \beta_{a} \gamma_{a}, \psi$ for $\delta_{a}, \beta_{a}, \psi_{a}$ respectively. In view of this observation, we can say that the analysis of this section provides new information as long as a segment specific goodwill decay parameter is present in the representation of the goodwill evolution.

After assuming as exogenous the product price $p=1$ and production cost function $c(\cdot)$, the optimal profit depends on the advertising medium features, i.e. the segment-spectrum $\left(\gamma_{a}\right)_{a \in A}$ and the productivity function $\varphi(\cdot)$. Then it is clear that the choice of a suitable advertising medium to reach the relevant market segments is crucial. In fact, easier selection of the best communication channels is a main reason for segmenting the market (see Kotler, 2003, p. 279). Moreover, we are led to consider the more general scenario in which a company allocates marketing resources to multiple media (Naik and Raman, 2003; Naik et al., 2005).

\section{Conclusion}

We have brought some market segmentation concepts into the statement of an advertising and production problem for a seasonal product with NerloveArrow's linear goodwill dynamics. To this purpose, we have followed the lines of some recent analyses concerning the introduction of a new product and the advertising process for sales over an infinite horizon. In today's highly competitive markets, it is important to know how much, when and also towards whom to advertise, especially in the case of seasonal products.

We have considered two kinds of situations. In the first one, the advertising process can reach selectively each segment. In the second one, only one advertising medium is available and it has a known effectiveness spectrum over the segment set. In either case we have formulated the general optimal control problem and have obtained the unique optimal solution, up to a parameter which is characterized as the solution of a numerical equation (transversality condition). In particular we have studied the optimal control problems in which goodwill productivity of advertising is concave, and good production costs are convex and quadratic and we have obtained the explicit optimal solutions.

We observe that the optimal advertising effort, or each component of it, decreases and eventually must vanish, because only the season profit has been taken into consideration as the objective. In particular, if the marginal productivity of a medium activation intensity is unbounded, then 
the optimal advertising effort reaches zero just at the final time; on the contrary, if the marginal productivity is bounded, then it is optimal to stop advertising before the end of the sale period.

Further directions of analysis should consider, on the one hand, the joint use of several advertising media which show different effectiveness features over the segment set and, on the other hand, the competition of different companies. A crucial aspect in the former extension is that of modelling the joint effect of the different media on each segment goodwill evolution, which must account for synergy of the multiple advertising efforts. As for the latter extension, the natural framework for the analysis of competition is that of differential games and a special phenomenon which must be considered in the model is the interference among the advertising actions of competing companies.

Acknowledgements We wish to thank the referees for their valuable comments and the editor Frank Plastria for his kind and effective attention to our requests: the presentation of the paper has been considerably improved by their contribution.

\section{References}

Buratto, A., Grosset, L., and Viscolani, B. (2006a). Advertising a new product in a segmented market. European Journal of Operational Research, 175:1262-1267.

Buratto, A., Grosset, L., and Viscolani, B. (2006b). Advertising channel selection in a segmented market. Automatica, 42:1343-1347.

Favaretto, D., Mazzega, P., Mezzaroba, A., and Viscolani, B. (1996). Optimal control of advertising and production of a seasonal product with nondifferentiable demand. Dynamics and Control, 6:91-104.

Favaretto, D. and Viscolani, B. (1997). Determining optimal production and advertising policies for a seasonal product. Optimization, 42:359-378.

Favaretto, D. and Viscolani, B. (1999). A multiperiod production and advertising problem for a seasonal product. Annals of Operations Research, 88:31-45.

Favaretto, D. and Viscolani, B. (2004). A general model for the marketing of seasonal products. Journal of Interdisciplinary Mathematics, 7(3):349-366.

Feichtinger, G., Hartl, R. F., and Sethi, S. P. (1994). Dynamic optimal control models in advertising: recent developments. Management Science, 40(2):195-226.

Grosset, L. and Viscolani, B. (2008). Advertising in a segmented market: comparison of media choices. IMA Journal of Management Mathematics, 19:219-226.

Jørgensen, S. (1982). A differential games solution to a logarithmic advertising model. Journal of the Operational Research Society, 33:425-432.

Jørgensen, S., Kort, P. M., and Zaccour, G. (2006). Advertising an event. Automatica, 42(8):1349-1355.

Jørgensen, S., Taboubi, S., and Zaccour, G. (2001). Cooperative advertising in a marketing channel. Journal of Optimization Theory and Applications, 110(1):145-158.

Jørgensen, S. and Zaccour, G. (2004). Differential Games in Marketing. Kluwer Academic Publishers, Boston.

Kotler, P. (2003). Marketing Management, 11th Edition. Prentice-Hall, Upper Saddle River.

Kotler, P. and Trias de Bes, F. (2003). Lateral Marketing: New Techniques for Finding Breakthrough Ideas. Wiley, Hoboken.

Naik, P. A. and Raman, K. (2003). Understanding the impact of synergy on multimedia communications. Journal of Marketing Research, 40(4):375-388. 
Naik, P. A., Raman, K., and Winer, R. S. (2005). Planning marketing-mix strategies in the presence of interactions. Marketing Science, 24(1):25-34.

Nerlove, M. and Arrow, K. J. (1962). Optimal advertising policy under dynamic conditions. Economica, 29(2):129-142.

Seierstad, A. and Sydsaeter, K. (1987). Optimal Control Theory with Economic Applications. North-Holland, Amsterdam.

Sethi, S. P. (1975). Optimal control of a logarithmic advertising model. Operational Research Quarterly, 26:317-319.

Weber, T. A. (2005). Infinite-horizon optimal advertising in a market for durable goods. Optimal Control Applications and Methods, 26(6):307-336. 